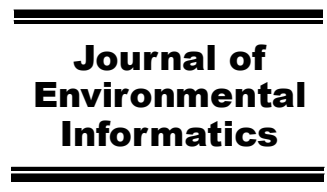

www.iseis.org/jei

\title{
Comparing the Radiometric Behavior of LISS III Data of IRS 1D and IRS P6 for Landscape Ecological Application
}

\author{
A. Sharma* \\ Agricultural \& Food Engineering Department, Indian Institute of Technology, Khragpur 721302, India \\ Received 14 July 2008; revised 9 January 2009; accepted 23 January 2009; published online 12 March 2009
}

\begin{abstract}
In the recent time, the usage of multi date satellite remote sensing data is a commonest practice to monitor and model the landscape dynamics. But the reliability of information obtained from such time series analysis of satellite imagery depends heavily on the coherence in the spectral behavior of the multi-dates satellite data. The inconsistency in the spectral behavior among the multidate satellite data may be attributed to differences in satellite orbit and sensor characteristics and even the environmental conditions. Indian Remote Sensing satellite (IRS) 1D and IRS P6 have been planned to have sensors of almost identical specifications to cater continued data service and to facilitate temporal analysis for resource planning and management. In the present paper an attempt has been made to compare the data quality of the Linear Imaging Self scanning System III (LISS III) data from IRS 1D and IRS P6 satellite of identical characteristics and to ascertain their usability for temporal analysis. The radiometric characteristics of the LISS III images of the closest dates from IRS 1D and IRS P-6 satellites were compared band wise. Spectral classes obtained through unsupervised clustering were used to compute several class level and landscape level metrics. Statistical analysis of radiometric and landscape characteristics revealed a significant difference in the investigated dataset and their usability for temporal analysis is subjected to proper calibration of radiometric behavior of the two data sources.
\end{abstract}

Keywords: Indian remote sensing satellite, landscape analysis, radiometric behavior, spectral cluster

\section{Introduction}

Remote sensing technology has made a significant progress for earth observation in last two decades. By virtue of its cost effectiveness, repetitive, multi-spectral and synoptic view has high appreciation in resource planning and management at landscape level. In landscape ecology remote sensing data is used for thematic mapping, resource inventory, change detection, spatial modeling, generation of management plans etc. To understand certain natural and man-made changes in landscape, a time step of 5 to 10 years or more is required (Nunes and Auge, 1999). The understanding of driving forces and rate at which the changes are occurring also require analysis of time series remote sensing data from one or other sensors. But the reliability of information obtained from such time series analysis of satellite imagery depends heavily on the coherence in the spectral behavior of the multi-dates satellite data. The inconsistency in the spectral behavior among the multi-date satellite data may be attributed to differences in satellite orbit characteristics and sensor characteristics such as spatial, radiometric, spectral resolutions and snag in sensor itself. Besides,

${ }^{*}$ Corresponding author. Tel.: +91 9832 749138; fax: +91 3222282244.

E-mail address: arbind_78@rediffmail.com (A. Sharma).

ISSN: 1726-2135 print/1684-8799 online

(C) 2009 ISEIS All rights reserved. doi:10.3808/jei.200900141 environmental factors such as atmospheric and illumination condition also imparts discrepancy in the spectral behavior of multi-dates satellite data (Jayabharathi et al., 2003).

In time-series data analysis of remote sensing data, spectral response of particular object with precise geometric fidelity at different time is very critical and is a key to success (Lunetta, 1999). Variation in solar illumination conditions, atmospheric absorption and scattering can affect detector performance by changing the receiving radiance value through addition to or subtraction from the expected radiance value (Jensen, 1996). Use of multi-date or multi-senor data for quantitative change detection studies require that either radiance measured in various bands by different sensors or conversion factors be known amongst them (Singh, 1989). This fact stimulate researcher to study inter-sensor radiometric behaviour and the corresponding band calibration to improve the conjunctive use of data from different sensors or data of different dates from same sensor. Robino (1982) has attempted the computation of physical values from Landsat digital data of Landsat 1, 2, 3 and 4 . The study revealed the significance of physical values especially when internally non-consistent data of different satellite/sensors are being used. Krishnamurthy et al. (1991) compared the radiometric behavior of IRS (Indian Remote sensing Satellite) 1A LISS (Linear Imaging Self-Scanning System) I and LISS II sensors by converting the digital number to radiance value. Through linear curve fitting and correlation coefficient they revealed good agreement between the correspond- 
ing bands of both the sensors. Jayabharathi et al. (2003) also reported similar results for sensors of IRS IC and IRS 1D satellite. Thome et al. (1997) and Teillet et al. (2000) provided cross calibration procedure for different sensors of LANDSAT satellite. Recent work in this direction includes comparison of the IRS-P6 and the Landsat sensors by Chander et al. (2008), intersensor calibration (Pasule et al., 2008; Senthilkumar et al., 2006), effect of radiometric uncertainty in vegetation analysis (Vicente-Serrano et al., 2008; Miura et al., 2000), burn severity detection (Wagtendonk et al., 2004), leaf area index calculation (Soudani et al., 2006), marine aerosol modeling (Mishra et al., 2008).

Indian Space Research Organization (ISRO) has launched IRS P6 in October 17, 2003 with the objectives of providing continuity to existing data from IRS 1D (launched in 1998) and to facilitate time series analysis. It also offers newer applications owing to enhanced capabilities of the sensors. The spatial and spectral resolution of LISS III sensor which onboard both IRS 1D and IRS P6 makes these data highly suitable in analyzing changes in vegetation cover and monitoring environmental processes. Although the dataset have identical parameter with pre launch calibration, may still have anomalous radiometric behavior due to any of the reasons viz., the detector aging, satellite injection vibration, onboard electronic system anomalies, orbital characteristics etc (Oza, 2004). It is, thus, imperative to carry out a comparative study of these dual sensors to ascertain the feasibility of using data in conjunction for a given application. None of the studies have analyzed the role of radiometric behavior of multitemporal LISS III data from these satellites for landscape dynamics.

The present research communications will fill that gap by considering a case study of landscape analysis along with the radiometric comparison of LISS III data from IRS 1D and IRS P6 satellite. The present work is aimed at a comparative appraisal of radiometric behavior of the LISS III data of IRSP6 and IRS-1D to ascertain the feasibility of using LISS III of the IRS 1D and IRS P6 in continuity and/or conjunction with each other for landscape analysis.

\section{Methodology}

\subsection{Study Area and Data Used}

Due to the limited number of co-incident image pairs between these sensors, the scene selection for these studies proved to be a challenge. Due to the lack of near-simultaneous images, traditionally required for sensor calibration and application evaluation, alternatively closest dates images that have high reflectance, large dynamic range, high spatial uniformity, high Sun elevation, and minimal cloud cover were investigated. The present study was carried out in Daltonganj district of Jharkhand state of India. The study area lies between $24^{\circ} 00^{\prime}$ to $24^{\circ} 15^{\prime} \mathrm{N}$ altitude and $84^{\circ} 15^{\prime}$ to $84^{\circ} 30^{\prime} \mathrm{E}$ longitude, covered by Survey of India (SOI) 72-D4 topographic map.

The final LISS III scenes selected for the current work were cloud-free scene from both satellite and covering part of the same footprint were procured from National Remote Sen- sing Centre (NRSC), Hyderabad. The detail characteristics of the sensor and satellites are presented in Table 1.

Table 1. Characteristics of LISS III Sensor of IRS 1D and IRS P6

\begin{tabular}{lll}
\hline Characteristics & IRS P 6 & IRS 1D \\
\hline Altitude & $817 \mathrm{~km}$ & $817 \mathrm{~km}$ \\
No. of orbit & 341 & 341 \\
Inclination & 98.7 & 98.53 \\
No of orbit/day & 14.203 & 14 \\
Orbit period (min) & $101.35 \mathrm{~min}$ & $101.35 \mathrm{~min}$ \\
Repetivity & 24 days & 24 days \\
Equatorial crossing time (Hr) & $10: 30 \pm 5$ & 10.30 to 10.47 \\
Date of Pass & 10.03 .2004 & 07.03 .2004 \\
Path/Row $\quad$ B1 (Green) & $104 / 055$ & $104 / 055$ \\
Spectral $\quad 0.52-0.59$ & $0.52-0.59$ \\
Resolution B2 (Red) & $0.62-0.68$ & $0.62-0.68$ \\
\multicolumn{1}{c}{ B3 (NIR) } & $0.77-0.86$ & $0.77-0.86$ \\
B4 (SWIR) & $1.55-1.70$ & $1.55-1.70$ \\
Spatial resolution & $23.5 \mathrm{~m}$ & $23.5 \mathrm{~m}$ for B1, B2, \\
& & B3 \& 70 m for \\
Radiometric resolution & 7 bits & band B4 bits \\
\hline
\end{tabular}

Both the IRS P6 and IRS 1D satellites were planned to have almost identical specifications to cater continued data requirements with minute different in orbit inclination and equatorial crossing time while the LISS III sensors are also identical characteristics with only exception for the spatial resolution of band 4 i.e. $23.5 \mathrm{~m}$ for IRS P6 while $70 \mathrm{~m}$ for IRS 1D (NRSA, 2003).

\subsection{Method}

Sub-scene of the study area was extracted from LISS III of IRS-1D and IRS P6. LISS III data were geo-referenced using survey of India (SOI) topographic maps of 1:50000 scale of the same area. Image to image registration was performed for IRS 1D LISS III data using geo-referenced LISS III image of IRS P6 as reference. RMS error for registration was restricted to less than 0.3 pixels.

The radiometric behavior of the two scenes were compared using their band histogram, radiometric profiles and entropy content for individual band and the first principal component (PC 1) image. Histogram statistics such as minimum, maximum, mean, standard deviation and contrast (range) were computed for all the corresponding bands of the two data sets by using ERDAS Imagine software. In order to compare the radiometric profile of LISS III data from the two satellite digital numbers (DN) for each band were collected at thirty-eight (38) random points. DN is the radiance energy received by the sensor followed by quantization as per the radiometric resolution of the sensor. Scatter plot was also used to compare the radiometric behavior of the corresponding band of the two LISS III data set.

The concept of entropy from theory of information content (Shannon and Weaver, 1949) was used to measure the 
amount of spatial information in each band of the LISS III images. Entropy can be calculated using Equation 1:

$$
H=-\sum_{i=1}^{m}\left(P_{i} \cdot \log _{2} P_{i}\right)
$$

where, $P_{i}$ is probability of a cell being classified as class type $i$ and $m$ is number of classes. The value of entropy increases with increase in information content of a particular band and vice-versa. Entropy content of first principal component that used to have more than $95 \%$ of information of an LISS III image were also compared for both the LISS III data. Principal components analysis (PCA) is a method of data compression that allows redundant data to be compacted into fewer bands and are often more interpretable than the source data (Jensen, 1996). To compute a principal components transformation of satellite sensor data is implemented computationally using three steps (Eklundh and Singh, 1993):

(i) Calculation of either variance-covariance matrix (nonstandardized) or correlation matrix (standardized) of the satellite sensor data;

(ii) Computation of the eigenvalues and eigenvectors of the variance-covariance matrix or correlation matrix; and

(iii) Linear transformation of the satellite sensor data using the coefficients of the eigenvector matrix.

To explore the effect of radiometric behavior on spectral clustering, an unsupervised classification called Iterative SelfOrganizing Data Analysis Technique (ISODATA) was used. ISODATA Clustering is iterative in that it repeatedly performs an entire classification (outputting a thematic raster layer) and recalculates statistics (Tou and Gonzalez, 1974). The process begins with a specified number of arbitrary cluster means or the means of existing signatures, and then it processes repetitively, so that those means shift to the means of the clusters in the data. On the first iteration of the ISODATA algorithm, the means of $N$ clusters can be arbitrarily determined. After each iteration, a new mean for each cluster is calculated, based on the actual spectral locations of the pixels in the cluster, instead of the initial arbitrary calculation. Then, these new means are used for defining clusters in the next iteration. The process continues until there is little change between iterations (Swain, 1973).

Seven spectral classes were obtained for both the images using all the four bands and by excluding the band 4 (SWIR). The Kappa statistic was used to measure the agreement or consistency between two dataset. Kappa statistics is an index which uses an error matrix and compares the agreement against that which might be expected by chance. Kappa coefficient is more than an absolute value of agreement as it includes a correction for chance agreement. The Kappa statistics can be written as:

$$
\hat{K}=\frac{N \sum_{i=1}^{r} x_{i i}-\sum_{j=1}^{r} x_{i+} \cdot x_{+i}}{N^{2}-\sum_{i=1}^{r} x_{i+} \cdot x_{+i}}
$$

where $r$ is the number of columns/rows of the error matrix; $N$ is the total number of observations; $x_{i i}$ is the number of coincident observations (diagonal elements of the matrix); and $x_{i+}$ and $x_{+i}$ are the marginal totals for $i^{\text {th }}$ row and column respectively. The possible values range from +1 (perfect agreement) via 0 (no agreement above that expected by chance) to -1 (complete disagreement) (Jensen, 1996).

In the next step, both the clustered images were put into FRAGSTAT software for exploring the effect of radiometric behavior of LISS III data in landscape analysis through spectral clustering. Patch level metrics such as number of patch (NP), patch density (PD), perimeter-area fractal dimension (PAFRAC), interspersion-juxtaposition index (IJI) and cohesion (COHESION) were used. While at landscape level three extra metrics i.e. largest patch index (LPI), Contagion (CONTAG) and Shannon diversity index (SHDI) were calculated for comparing the structural and compositional pattern of the two clustered image generated from LISS III data of IRS 1D and IRS P6. For details on the landscape metrics used in the present study, one may refer to McGarigal and Marks (1995).

\section{Results and Discussions}

\subsection{Site Selection and Preprocessing}

The accuracy of the inter sensor comparison depends mainly on the uniformity of the site, positioning accuracy, viewing angle differences and uncertainties from atmospheric conditions. Due care has been taken in this direction during the set up of research work. The study site selected generally shows little seasonal variation and is relatively flat to avoid terrain induced distortion in image for different viewing angle. The time of image acquisition is the second week of March with three days interval when the atmospheric condition remains relatively stable over the study site. To keep the positional accuracy within admissible limit for inters sensor comparison, image-to-image registration was performed avoiding misregistration and overall RMSE was kept below 0.3 pixel.

LISS III sensor exhibits linear response to incoming radiance from the Earth's surface radiance. This response is quantized into 8-bit values that represent brightness values commonly called Digital Number (DN). DN value has been used for radiometric comparison of the sensors in the subsequent analysis because digital classification of remote sensing images is based on this physical number.

\subsection{Radiometric Comparison}

In the present study radiometric comparisons of corresponding bands of the two sensors were carried out using histogram statistics, radiometric profile, scatter plot and entropy. Ideally, both the LISS III data should be radiometrically identical due to almost similar sensor specification and unchanged environmental condition such as sun illumination, surface and atmospheric properties. However, the band wise histogram statistics of the two data presented in Table 2 reveals some anomalies. 
Table 2. Histogram Statistics of Different Bands of LISS III Data of IRS1D and IRS P6

\begin{tabular}{lllllllll}
\hline \multirow{2}{*}{$\begin{array}{l}\text { Histogram } \\
\text { statistics }\end{array}$} & \multicolumn{9}{c}{ IRS 1D LISS III } & \multicolumn{3}{c}{ IRS P 6 LISS III } \\
\cline { 2 - 8 } & Band1 & Band2 & Band3 & Band4 & Band1 & Band2 & Band3 & Band4 \\
\hline Min & 69 & 46 & 28 & 54 & 87 & 47 & 28 & 57 \\
Max & 203 & 189 & 160 & 255 & 255 & 220 & 186 & 255 \\
Mean & 117.37 & 104.39 & 100.49 & 213.77 & 152.71 & 115.72 & 118.68 & 171.01 \\
S.D & 14.36 & 16.87 & 11.98 & 22.43 & 18.45 & 19.3 & 14.49 & 20.43 \\
Contrast & 134 & 143 & 132 & 201 & 168 & 173 & 158 & 198 \\
\hline
\end{tabular}

The results show that the radiance values are not saturating for any data set. The DN values of the four bands of 1D LISS III are $117.37 \pm 14.36,104.39 \pm 16.87,100.49 \pm 11.98$ and $213.77 \pm 22.43$ respectively while the corresponding values for P6 LISS III are $152.71 \pm 18.45,115.72 \pm 19.30$, $118.68 \pm 14.49$ and $171.01 \pm 20.43$ respectively. It has been observed that the minimum, maximum, mean and contrast value of IRS P6 are comparatively higher than the corresponding value of IRS1D in all bands except band 4 (SWIR).

The higher DN value and contrast in the band 4 of LISS III data of IRS 1D due to fact that it receive radiance from a bigger land unit $(70 \mathrm{~m} \times 70 \mathrm{~m}$ as compared to $30 \mathrm{~m} \times 30 \mathrm{~m}$ in case of IRS P6) and hence the radiance converted DN value is higher than the IRS P6. Similarly, the standard deviation of DN for all band except band 4 (SWIR) is greater for IRS P6 LISS III than the IRS 1D LISS III. A broadening of the band histogram of P6 LISS III suggests improvement in dynamic range and information loads as compared to 1D LISS III. It further indicates that a better classification results would be obtained with IRS P6 LISS III data for given number of class. In case of P6 LISS III, radiometric saturation is obtained for band 1 (Green) and band 4 (SWIR) while in 1D LISS III saturation is obtained for band 4 only.

Theoretically, the radiation emitted by a target recorded simultaneously by the two independent sensors should be ideally same i.e. there should be 1:1 correspond between the their radiometric value in all the bands. For comparison of the two sensors, the band wise radiometric profile (Figures 1 to 4 ) and scatter plot for DN value of P6 LISS IIII vs 1D LISS III were presented in Figures 5 to 8 . A radiometric profile illustrates the spectral behavior of given band in a spatially distributed manner in contrast to global statistics discussed above and was obtained by recording the DN value at thirty eight (38) random points

The figures demonstrate a wide gap i.e. deviation between the radiometric profiles of the two sensors in all the bands. The deviations are more in case of band 1 and band 4. Despite of difference in DN value between the sensors, they follow a similar trend through out the sample point in all the four bands. The figure also reveals that the DN value of LISS III image of IRS P6 are consistently higher than that of IRS 1D in bands 1, 2, and 3 while for band 4 reverse is true. The higher contrast characteristics of all the four bands incase of IRS P6 ensure better quality for data display and interpretation.

The $\mathrm{R}^{2}$ and linear regression function for obtained by plo- tting the DN value corresponding band of P6 LISS III against 1D LISS along with plot are presented in Figures 5 to 8 . The expected 1: 1 reflectance line is also plotted for reference. $\mathrm{R}^{2}$ show the strength of linear relationship between the corresponding bands of investigated dataset and the regression function obtain through least squares fit gives the cross-calibration gain and biases as the coefficients of the linear fit. These coefficient can be used to cross calibrate the two sensor for the study site.

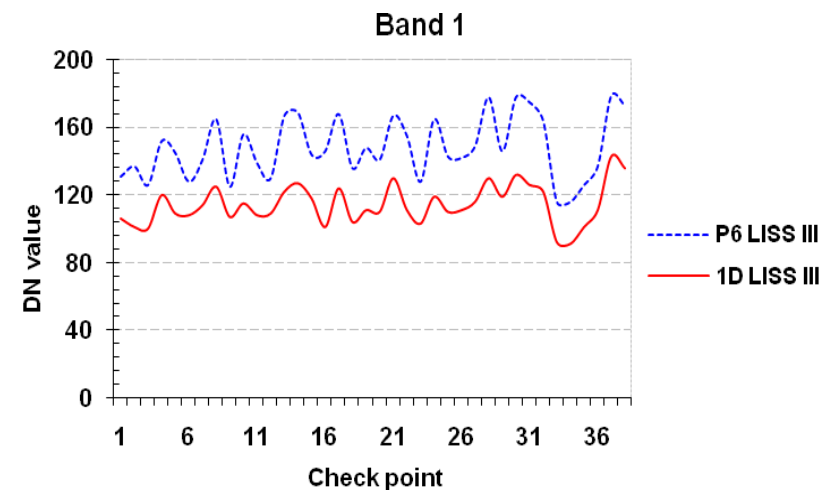

Figure 1. Radiometric profile of band 1 of IRS P6 LISS III and IRS 1DS LISS III.

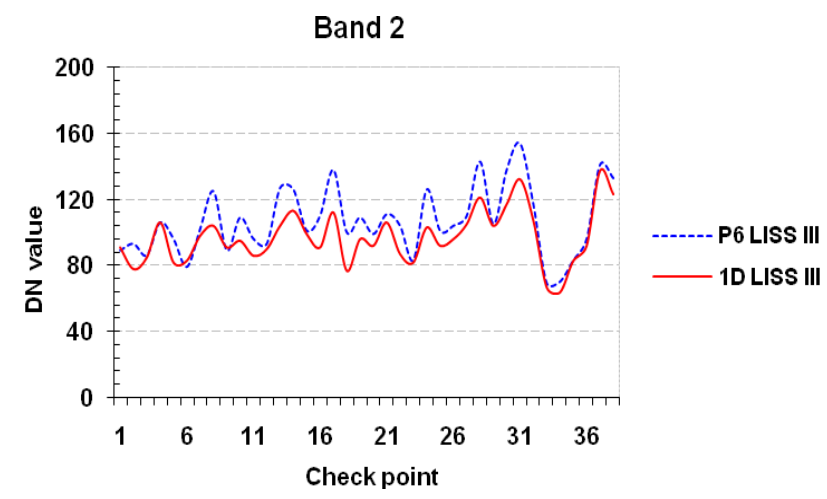

Figure 2. Radiometric profile of band 2 of IRS P6 LISS III and IRS 1DS LISS III.

The figures suggest a good correlation for Bands 1, 2 and 3 while there is a poor correlation $\left(\mathrm{R}^{2}=0.5171\right)$ for band 4 of the two sensors. The order of correlation among the different bands is in the order of Band $1>$ Band $2>$ Band $3>$ Band 4 . 
However, the figures show a systematic deviation from the one-to-one line, which is expected, in all the bands. Despite of good correlation among Bands 1,2 and 3 of the two sensors, plots are biased towards the P6 LISS III (above the 1:1 line) while for band 4 the plot is biased towards the 1D LISS III (below the 1:1 line). The magnitude of deviation is in the order of Band $4>$ Band $1>$ Band $3>$ Band 2. Thus it is the band two (high correlation and least deviation) for which both the sensors are most consistent in their radiometric behavior. In contrast, it is the band 4 (least correlation and highest deviation) followed by band 1 which are most significant contributors towards radiometric anomaly between the two sensors. It can also be seen from Figures 5 to 8 that the gap between the 1:1 line linear regression line widen up towards higher DN value i.e the sensor anomaly increases at high reflectance region or at near saturation reflectance for all the four bands.

\section{Band 3}

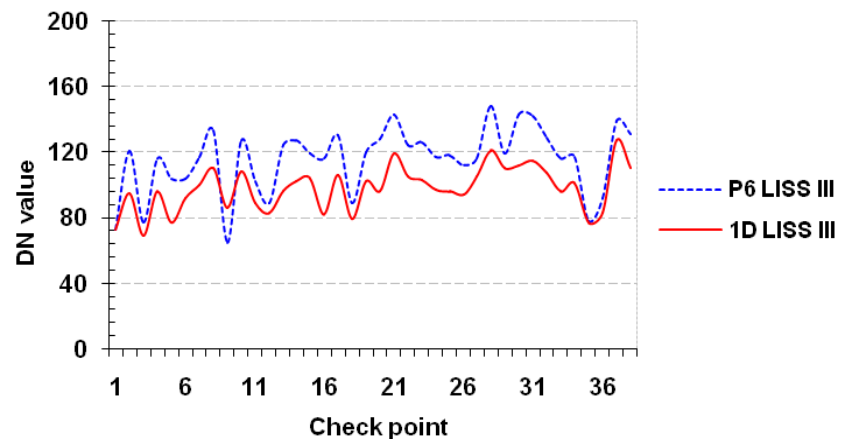

Figure 3. Radiometric profile of band 3 of IRS P6 LISS III and IRS 1DS LISS III.

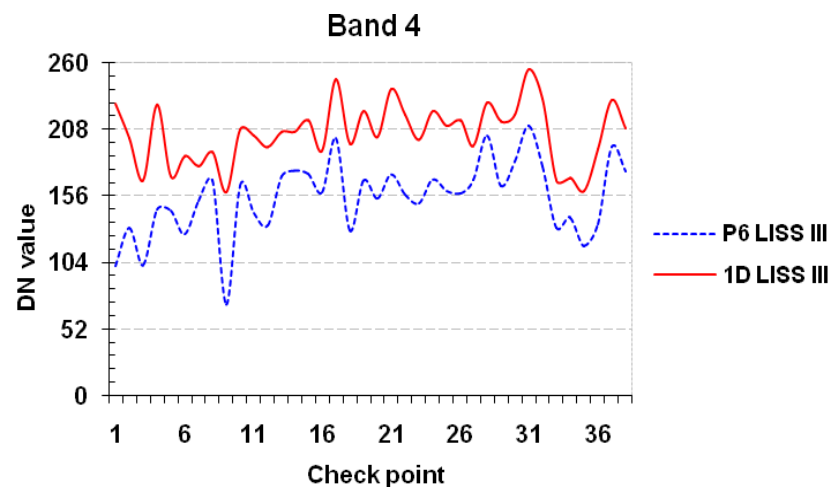

Figure 4. Radiometric profile of band 4 of IRS P6 LISS III and IRS 1DS LISS III.

The fact of relatively better radiometric quality of the P6 LISS III as compared to 1D LISS III has been further supported by comparing the information content or entropy measure of the individual band and first principal component of the LISS III images (Figure 9).

Entropy of a raster band describes the total information content in the image and higher entropy is desirable for better image interpretation. The Figure 5 clearly demonstrate that information content of first three bands and the first principal component (PC 1) of IRS P6 LISS III is higher than its corresponding value for IRS 1D LISS III. Entropy for Band 4 (SWIR) was not calculated as entropy for band 4 is deemed to be higher for IRS P6 due to higher spatial resolution. Within the sensor the entropy content were found to be in the order of PC $1>$ Band $2>$ band $1>$ Band 3 for both the sensors.

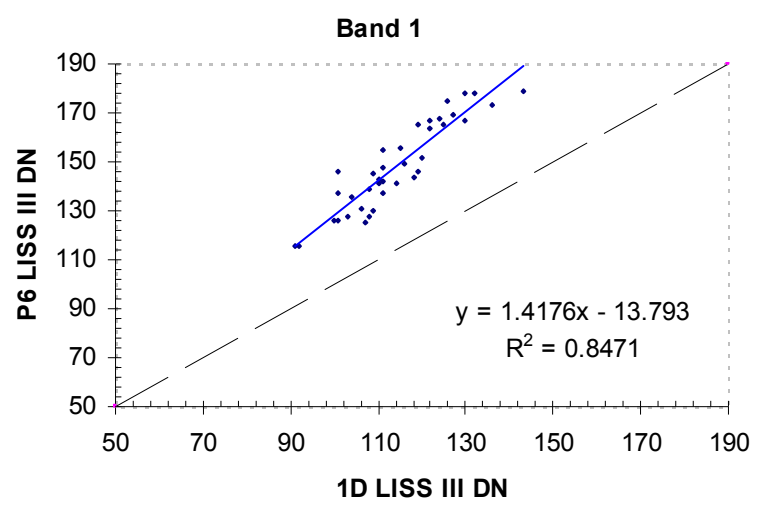

Figure 5. Regression line of DN value of band 1 of LISS-III images of IRS 1D and IRS P6.

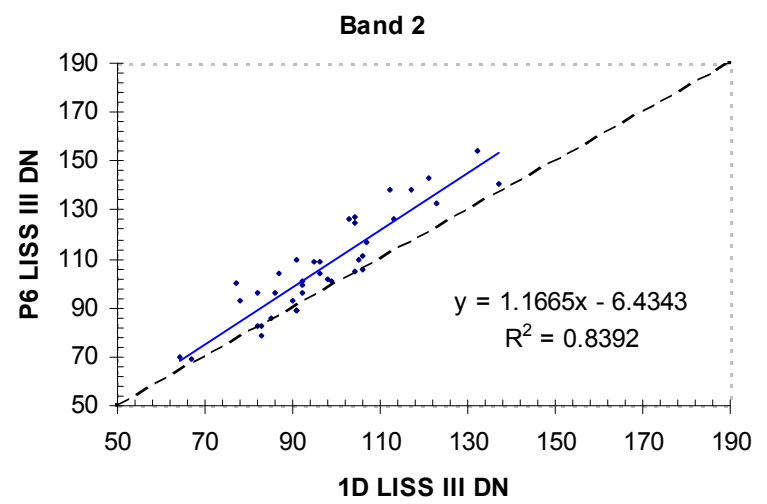

Figure 6. Regression line of DN value of band 2 of LISS-III images of IRS 1D and IRS P6.

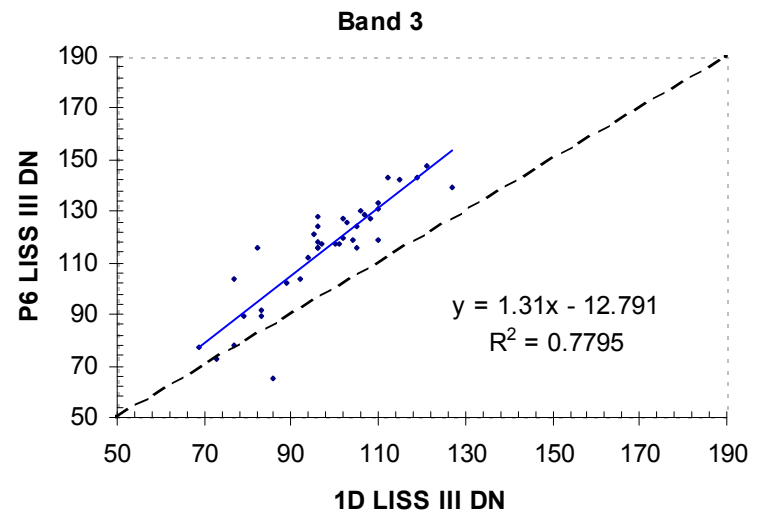

Figure 7. Regression line of DN value of band 3 of LISS-III images of IRS 1D and IRS P6. 


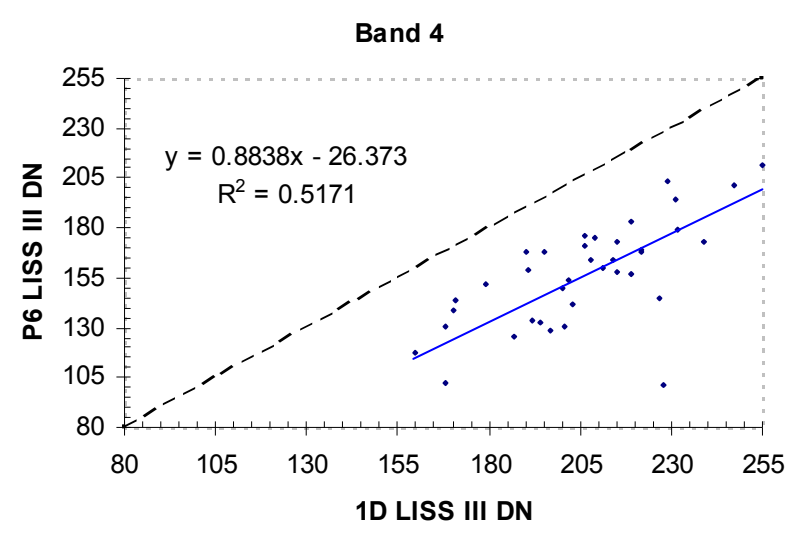

Figure 8. Regression line of DN value of band 4 of LISS-III images of IRS 1D and IRS P6.

\subsection{Unsupervised Classification}

From the preliminary radiometric comparison discussed above it is clear that band 4 is major source of uncertainty. Therefore, clusters were generated using all the bands as well by excluding the band 4 to check for any improvement in classification and landscape analysis. Moreover to avoid any user induced bias unsupervised classification was chosen. Thus, theoretically an identical clustering would be expected. However, the classification outputs presented in Table 3 indicate anomalous spectral clustering.

Table 3 shows a comparative distribution of number of pixel under each spectral class for both the data set using all band and by excluding the band 4 (SWIR). Examining clustered image generated using all bands, highest variation are observed for class $2(28.57 \%)$, class $1(10.05 \%)$ and class 7 $(10.5 \%)$ while lowest variation are observed for class $4(-0.97 \%)$ and class $3(-2.14 \%)$. On excluding the band 4 for cluster generation changes in the percentage difference and their signs are observed. It is quite apparent from Table 3 that the class 2 and class 1 are the highly affected clusters due to spectral anomalies but there is a decrease in their percentage of variation on excluding the band 4 in spectral clustering. But it is class 3 $(2.23 \%)$ instead of class $4(-7.49 \%)$ that show least amount of anomaly on excluding the band 4 in unsupervised classification. However, it is very difficult to interpret whether there is a improvement in consistency in spectral clustering on excluding the band 4.

In order to validate and cross check the consistency between the two dataset for spectral clustering Kappa statistics was used. During Kappa analysis the clustered images of IRS 1D and IRS P6 were used as reference and classified image respectively and the obtained results are presented in Table 4.

Table 4 reports the four accuracy statistics i.e. accuracy, omission error, commission error and KHAT. Accuracy describes the proportion of pixels that is classified into a same cluster class in both the classified images. Omission error is the proportion of pixels that is misclassified into cluster other than the cluster of interest while commission error is the proportion of pixels that is misclassified as cluster of interest. KHAT is the output of these three statistics after a correction has been applied for chance accuracy. The overall accuracy statistics presented in Table 4 demonstrate a little improvement in accuracy statistics on excluding the band 4 in cluster generation as envisaged from high value of overall accuracy and KHAT value. This improvement may be accounted to low correlation between the DN values of band 4 as compared to other bands of the two dataset being investigated. The other reasons may be the malfunctioning of band 4 of IRS 1D LISS III image as some bad strip or line is easily visible in its band 4 and difference in spatial resolution for the band 4 of the two sensors. The KHAT values of clustering using all band and excluding the band 4 are 0.3541 and 0.3572 respectively and in both the cases the p-value for KHAT are found to be $<$ 0.0001 at $95 \%$ confidence interval. The results imply that the two dataset may lead to significantly inconsistency in cluster

Table 3. Number of Pixels Under Each Spectral Class in Different Classified Images (in Thousands)

\begin{tabular}{|c|c|c|c|c|c|c|}
\hline \multirow{2}{*}{$\begin{array}{l}\text { Spectral } \\
\text { Class }\end{array}$} & \multicolumn{3}{|c|}{ B-1234 } & \multicolumn{3}{|c|}{ B-123 } \\
\hline & P6 & $1 \mathrm{D}$ & $\%$ variation & P6 & $1 \mathrm{D}$ & $\%$ variation \\
\hline 1 & 111.462 & 132.774 & -16.051 & 151.222 & 168.729 & -10.376 \\
\hline 2 & 199.431 & 155.113 & 28.571 & 177.330 & 149.045 & 18.977 \\
\hline 3 & 223.544 & 228.426 & -2.137 & 216.511 & 211.789 & 2.230 \\
\hline 4 & 273.211 & 275.883 & -0.969 & 264.375 & 285.786 & -7.492 \\
\hline 5 & 318.409 & 342.157 & -6.941 & 321.877 & 341.805 & -5.830 \\
\hline 6 & 255.004 & 240.509 & 6.027 & 255.112 & 233.208 & 9.392 \\
\hline 7 & 52.847 & 59.046 & -10.499 & 47.481 & 43.546 & 9.036 \\
\hline
\end{tabular}

Table 4. Overall Accuracy Statistics of Clustering Using All Bands and Excluding the Band 4

\begin{tabular}{lllll}
\hline Statistics & Accuracy & Omission Error & Commission Error & KHAT \\
\hline All bands & 0.4627 & 0.5373 & 0.0895 & 0.3541 \\
Band-123 & 0.4669 & 0.5331 & 0.0888 & 0.3572 \\
\hline
\end{tabular}


generation if proper calibration is not applied.

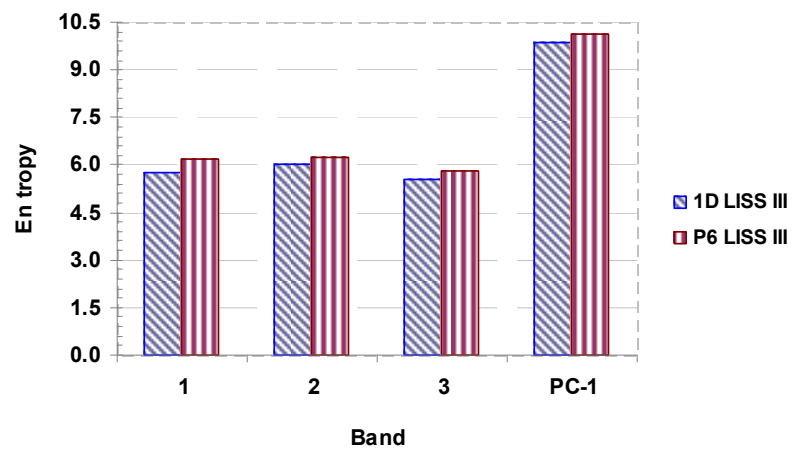

Note: 1, 2, 3 and PC-1 represents bands 1, 2, and 3 and first principal component derived using all the four bands.

Figure 9. Entropy of first three bands and first principal component (PC-1) of LISS-III images of IRS 1D and IRS P6.

\subsection{Landscape Analysis}

Landscape metrics are a standard tool in the study and monitoring of landscape pattern and change. But their calculation is highly sensitive to the input classisfied thematic map which in turn significantly affected by the radiometric characteristics of the remote sensing data. The results of landscape analysis in the present study also confirmed siginificant vari- ation in patch level and landscape level metric computed from LISS III image of IRS P6 and IRS 1D satellite that have otherwise identical specification. As planned, to explore the impact of radiometric inconsistency in the two dataset on landscape analysis through spectral clustering, various landscape metrics were calculated at class level using Fragstat software.

Table 5 shows the percentage of deviation in class level metric derived from clustered image generated using all the bands as well as by excluding the band 4 of both the sensor. The deviation was calculated by subtracting the class level metric value obtained from 1D LISS III image from its corresponding value obtained from P6 LISS III image. It is quite obvious from the Table 5 that high amount of deviation in NP, PD, and IJI for all the spectral classes while least in PAFRAC in both cases. However on excluding the band 4 in clustering, there is a considerable improvement i.e. decrease in deviation of class level metric for all the cluster classes except cluster class 7 .

Table 6 gives an idea about the landscape level metric calculated from both the images using all the bands and by excluding band 4 . The table also show percentage deviation in the value of landscape metric calculated from P6 LISS and 1D LISS III image. It is evident from the high value of NP and PD for the LISS III data of IRS P6 that it creates a comparatively more patchy pattern for the study site. These patchy pattern cause intermingling of different patch type and made the landscape more complex as these are obvious from the com-

Table 5. Percentage Deviation in Different Class Level Metrics Obtained from Two Sensors Using All the Bands and by Excluding Band 4

\begin{tabular}{|c|c|c|c|c|c|c|c|c|c|c|}
\hline \multirow[t]{2}{*}{ Class } & \multicolumn{5}{|c|}{ Percentage Deviation-all band } & \multicolumn{5}{|c|}{ Percentage Deviation-excluding band 4} \\
\hline & NP & PD & PAFRAC & IJI & COHESION & $\mathrm{NP}$ & PD & PAFRAC & IJI & COHESION \\
\hline 1 & 78.53 & 78.51 & 5.24 & 51.09 & -0.30 & 35.28 & 35.22 & 1.54 & 41.58 & -0.22 \\
\hline 2 & 60.99 & 60.95 & 1.25 & 25.46 & -1.21 & 29.89 & 29.83 & -0.69 & 10.65 & -1.19 \\
\hline 3 & 51.83 & 51.79 & -1.16 & 21.49 & -14.54 & 20.81 & 20.74 & -0.88 & 12.03 & -8.00 \\
\hline 4 & 54.69 & 54.66 & -1.59 & 22.59 & -18.62 & 18.50 & 18.43 & -1.57 & 12.03 & -8.16 \\
\hline 5 & 70.58 & 70.56 & -0.45 & 11.07 & -14.36 & 25.53 & 25.47 & -0.87 & 10.61 & -9.15 \\
\hline 6 & 64.92 & 64.89 & 2.67 & 30.83 & -3.57 & 28.43 & 28.37 & 0.77 & 18.01 & -1.46 \\
\hline 7 & 34.55 & 34.49 & 0.74 & 28.56 & -3.02 & 45.26 & 45.21 & 0.83 & 26.94 & -3.01 \\
\hline
\end{tabular}

Note: $\mathrm{NP}=$ number of patch, $\mathrm{PD}=$ patch density, PAFRAC $=$ perimeter area fractal dimension, IJI = interspersion juxtaposition index

Table 6. Different Landscape Level Metrics Obtained from Thematic Maps Generated by Using all the Four Bands and by Excluding the Band 4

\begin{tabular}{lllllll}
\hline Landscape & \multicolumn{3}{c}{ Band-1234 } & \multicolumn{2}{c}{ Band-123 } \\
\hline Metric & P6 LISS-III & 1D LISS III & \% change & P6 LISS-III & 1D LISS III & \% change \\
NP & 184738 & 74364 & 59.746 & 202587 & 153962 & 31.582 \\
PD & 232.749 & 93.771 & 59.711 & 255.237 & 194.143 & 31.469 \\
LPI & 2.791 & 2.371 & 15.033 & 4.127 & 4.592 & -10.129 \\
PAFRAC & 1.404 & 1.406 & -0.121 & 1.404 & 1.415 & -0.785 \\
CONTAG & 24.705 & 32.273 & -30.632 & 24.065 & 27.748 & -13.276 \\
IJI & 70.998 & 62.330 & 12.209 & 71.333 & 65.444 & 8.998 \\
COHESION & 90.893 & 95.205 & -4.744 & 89.773 & 91.546 & -1.937 \\
SHDI & 1.841 & 1.843 & -0.098 & 1.848 & 1.834 & 0.774 \\
\hline
\end{tabular}

Note: $\mathrm{NP}=$ number of patch, $\mathrm{PD}=$ patch density, $\mathrm{LPI}=$ largest patch index, PAFRAC $=$ perimeter area fractal dimension, $\mathrm{CONT}=$ contagion, $\mathrm{IJI}=$ interspersion juxtaposition index, SHDI $=$ Shannon diversity index 
paratively high value of IJI and low value of COHESION for P6 LISS III image generated landscape metric in both the cases. The iregularity in the values of the rest of the metric constrained to generalize any specific trend in them. The $\mathrm{p}$-values of paired t-test for the two dataset in both case are found to be 0.35 . The medium p-value of landscape level metric again indicates a lack of coherence between the dataset. The important observation is that on excluding the band 4 there is a considerable decrease in percentage deviation of the all metrics but PAFRAC and SHDI calculated using the LISS III imaged from the two investigated satellite i.e. IRS P6 and IRS 1D.

\section{Conclusions}

Environmental modelers frequently use remote sensing data from different satellites in order to obtain a cloud-free image, complete coverage of the study site or time series analysis over an extended period of time. However, each remotely sensed satellite image source has its own specifications which raise the issue of the feasibility or compatibility of using radiometric data acquired from different sensors. In the present study the radiometric behavior of LISS III data from IRS 1D and IRS P6 compared and their consequences on landscape analysis through spectral clustering was presented. The study has also revealed the significance of physical values (DN value) which are the basis of digital classification. The promising results of the presented study may open new perspectives on spatial and temporal use of multi-sensor data for landscape analysis. Results from different radiometric analysis, spectral clustering and landscape analysis show moderate agreement between the two dataset that were not radiometrically calibrated. However, the radiometric behavior of IRS P6 data is better than that of IRS 1D as envisaged from improved spatial resolution for band 4 , high contrast in histogram analysis and entropy of the individual band and PC 1. The results of spectral clustering and the subsequent test for coherence using various accuracy statistics and landscape analysis show a statistically significant inconsistency in the investigated dataset. The various band wise radiometric analysis identified band 4 (SWIR) as main source of uncertainty in using the LISS III image from IRS 1D and IRS P6. This inference has been further strengthen by the increase in KHAT value in kappa analysis of the clustered image and reduction in the percentage deviation of the both patch as well class level metrics on excluding the band 4 in analysis. Thus, it is advisable to perform band wise radiometric calibration between two sensors whenever data from different satellites/ sensors or multi-date data from same sensor are to be used in conjunction to obtain a more reasonable and reliable result.

\section{References}

Chander, G., Coan, M.J., and Scaramuzza, P.L. (2008). Evaluation and Comparison of the IRS-P6 and the Landsat Sensors, IEEE Trans. Geosci. Remote Sens., 46(1), 209-221, doi:10.1109/TGRS.2 007.907426.

Eklundh, L., and Singh, A. (1993). A comparative analysis of stan- dardised and unstandardised principal component analysis in remote sensing, Int. J. Remote Sens., 14 (7), 1359-1370, doi:10.1080 /01431169308953962.

Jayabharathi, S., Jayachandraiah, B., Muralikrishnan, S., and Senthil Kumar, A. (2003). Cross-calibration of IRS 1C and IRS 1D: WIFS Using near Synchronous Matching Scene. Proceeding of 24th Annual Conference of Indian Society of Remote Sensing, Thiruvananthapuram, 24-33.

Jensen, J.R. (1996). Introductory digital image processing: A remote sensing perspective (2nd ed.), New Jersey. Prentice - Hall.

Kirshnamurthy, J., Padmavathy, A.S., Jayaraman, V., and Uday, R. (1991). Satellite - 1A Linear Imaging Self Scanning Sensors for Radiometric behavior, Proceeding of 12th Asian Association of remote Sensing, Singapore, 65-71.

Lunetta, R.S. (1999). In: Lunetta, R.S., Elvidge, C.D. (Eds.), Remote Sensing Change Detection; Environmental Monitoring Methods and Applications, Taylor \& Francis, London, pp. 318.

McGarigal, K., and Marks, B.J. (1995). FRAGSTATS: spatial pattern analysis program for quantifying landscape structure. Gen. Tech, Report PNW-GTR-351, USDA Forest Service, Pacific Northwest Research Station, Portland.

Mishra, A.K., Dadhwal, V.K., and Dutt, C.B.S. (2008). Analysis of marine aerosol optical depth retrieved from IRS-P4 OCM sensor and comparison with the aerosol derived from SeaWiFS and MODIS sensor, J. Earth System Science, 117, 361-373.

Miura, T., Huete, A.R., and Yoshioka, H. (2000). Evaluation of Sensor Calibration Uncertainties on Vegetation Indices for MODIS, IEEE Trans. Geosci. Remote Sens., 38(3), 1399-1409, doi:10.1109/ 36.843034 .

NRSA (2003). Resourcesat-1 (IRS-P6) Data User's Handbook, Publication No. IRS-P6/NRSA/NDC/HB-10/03, National Remote Sensing Agency, Hyderabad, India.

Nunes, C., and Auge, J.I. (1999). Science, objectives and scope of LUCC, Land-Use and Land-Cover changes implementation strategy, IGBP Report 48, 122-124.

Palsule, S.S., Shah, B., Paswan, G., Sharma, A., Garg, A., Pandy, H., Srivastava, S.S., and Roy, S. (2008). Resourcesat-1 data quality evaluation system, Int. J. App. Earth Obs. Geoinf., 10, 147-158, doi:10.1016/j.jag.2008.02.004.

Oza, M.P. (2004). IRS-P6 Early Evaluation Studies, Scientific Report SAC/RESIPA/SR, October 02, 2004, 1-2.

Robino, C.J. (1982). Computation with physical values from Landsat data, Photogramm. Eng. Remote Sensing, 48(5), 781-784.

Senthilkumar, A., Srinvas, V., Jayabharathi and Muralikrishnan, S. (2006). Cross-calibration of IRS-1C, IRS-1D and IRS-P6: wide angle sensors using synchronous or near synchronous matching scenes, In: Accepted paper for the ISPRS Commission IV, WG IV/10 Symposium, Goa, India, September, 27-30.

Shannon, C., and Weaver, W. (1949). The mathematical theory of communication, University of Illinois Press, Urbana.

Singh, A. (1989). Review article - digital change detection techniques using remotely sensed data, Int. J. Remote Sensing, 10(6), 9891003.

Soudani, K., François, C., Maire, G., Dantec,V., and Dufrêne, E. (2006). Comparative analysis of IKONOS, SPOT, and ETM+ data for leaf area index estimation in temperate coniferous and deciduous forest stands, Remote Sens. Environ., 102, 161-175.

Swain, P.H. (1973). Pattern Recognition: A Basis for Remote Sensing Data Analysis (LARS Information Note 111572), West Lafayette, Indiana: The Laboratory for Applications of Remote Sensing, Purdue University.

Teillet, P.M., Markham, B.L., Barker, J.L., Storey, J.C., Irish, R.R., and Seiferth, J.C. (2000). Landsat Sensor Cross-Calibration Using Nearly-Coincident Matching Scenes, Proceedings of SPIE, vol. 4049, 155-167. 
Thome, K., Markham, B., Barker, J., Slater, P., and Biggar, S. (1997). Radiometric Calibration of Landsat, Photogramm. Engg. Remote Sens., 63(7), 853-858.

Tou, J.T., and Gonzalez, R.C. (1974). Pattern Recognition Principles, Reading, Massachusetts: Addison-Wesley Publishing Company.

Vicente-Serrano, S.M., Pérez-Cabello, F., and Lasanta, T. (2008). Assessment of radiometric correction techniques in analyzing ve- getation variability and change using time series of landsat images, Remote Sens. Environ., 112, 3916-3934, doi:10.1016/j.rse.2008.06. 011.

Wagtendonka, J.W., Rootb, R.R., and Key, C.H. (2004). Comparison of AVIRIS and Landsat ETM+ detection capabilities for burn severity, Remote Sens. Environ., 92, 397-408, doi:10.1016/j.rse.2003.1 S2.015. 